\title{
sciendo
}

\section{CHARACTERIZATION OF THREE GENERATIONS OF TRANSGENIC PIGS EXPRESSING THE HLA-E GENE*}

\author{
Magdalena Hryhorowicz ${ }^{1}$, Joanna Zeyland ${ }^{1}$, Agnieszka Nowak-Terpiłowska ${ }^{1}$, Jacek Jura ${ }^{2}$, \\ Wojciech Juzwa ${ }^{3}$, Ryszard Słomski ${ }^{1,4}$, Jan Bocianowski ${ }^{5}$, Zdzisław Smorąg ${ }^{2}$, Anna Woźniak ${ }^{6}$, \\ Daniel Lipiński ${ }^{1 *}$ \\ ${ }^{1}$ Department of Biochemistry and Biotechnology, Poznan University of Life Sciences, \\ Dojazd 11, 60-632 Poznań, Poland \\ ${ }^{2}$ Department of Animal Reproduction, National Research Institute of Animal Production, \\ 32-083 Balice n. Kraków, Poland \\ ${ }^{3}$ Department of Biotechnology and Food Microbiology, Poznan University of Life Sciences, \\ Wojska Polskiego 48, 60-627 Poznań, Poland \\ ${ }^{4}$ Institute of Human Genetics, Polish Academy of Sciences, Strzeszyńska 32, \\ 60-101 Poznań, Poland \\ ${ }^{5}$ Department of Mathematical and Statistical Methods, Poznan University of Life Sciences, \\ Wojska Polskiego 28, 60-637 Poznań, Poland \\ ${ }^{6}$ The NanoBioMedical Centre, Adam Mickiewicz University, Umultowska 85, \\ 61-614 Poznań, Poland \\ •Corresponding author: lipinskidaniel71@gmail.com
}

\begin{abstract}
The use of pigs as a source of organs and tissues for xenotransplantation can overcome the growing shortage of human donors. Human NK cells play an important role in the cell-mediated rejection of pig-to-human xenografts. In this paper we report the generation and extensive characterization of three generations of transgenic pigs with HLA-E gene encoding the antigen which can inhibit the human NK cell-mediated response. The gene construct pHLAE-GFPBsd containing the human gene encoding the human leukocyte antigen under the promoter of the EF-1 $\alpha$ elongation factor ensuring systemic expression was introduced by microinjection into a pronucleus of the fertilized porcine oocyte. PCR analysis revealed and FISH analysis confirmed that the pHLAEGFPBsd gene construct was present in the genome of the founder female pig. As a result of interbreeding, an additional 7 transgenic animals were obtained (one individual from $F 1$ generation and six individuals from F2 generation). The transgene expression was shown by RT-PCR and flow cytometry. Real Time PCR analysis estimated the approximate number of transgene copies at 16-34. Karyotype analysis did not show any changes in the structure or the number of chromosomes. The expression level of the transgene was stable in the next generation of genetically modified pigs. An NK cell-mediated cytotoxicity assay showed the increased viability of the trans-
\end{abstract}

*This work was supported by The National Centre for Research and Development (Grant No. INNOMED/I/17/NCBR/2014) in the framework of the "INNOMED" programme titled "Development of an innovative technology using transgenic porcine tissues for biomedical purposes". Acronym: "MEDPIG". 
genic cells in comparison with the wild-type, which confirmed the protective influence of HLA-E expression.

Key words: transgenic pigs, transgene expression, xenotransplantation, transgene stability, transgene inheritance

Xenotransplantation, the transplantation of living cells, tissues or organs from one species to another, could solve the problem of human organ shortages. Currently, the domestic pig (Sus scrofa) is considered the best donor of biological material for xenotransplantation (Prather et al., 2008). A significant cause of delayed and acute xenotransplant rejection is connected with reactions involving human NK cells (Puga Yung et al., 2017). The susceptibility of porcine endothelial cells to lysis triggered by human NK cells is caused by the failure of the inhibitory receptors of human NK cells to recognize porcine major histocompatibility complex (MHC) class I molecules. This is due to differences within the MHC of pigs (swine leukocyte antigens, SLA) and of humans (human leukocyte antigens, HLA) (Sullivan et al., 1997). The introduction of the human HLA-E gene into the porcine genome can be used to activate NK cell inhibitory receptors and inhibit the human NK cell-mediated response. It was shown that the insertion of the HLA-E gene into the porcine genome partially protects porcine endothelial cells in vitro against damage (Lilienfeld et al., 2007; Weiss et al., 2009). Moreover, Maeda and co-workers demonstrated that the insertion of the HLA-E gene into the pig genome protects porcine endothelial cells not only against human NK cell cytotoxicity, but also against macrophage-mediated cytotoxicity. The analysis of inducible nitric oxide (NO) synthase showed that transgenic expression of HLA-E suppressed the production of NO, indicating that HLAE can reduce macrophage cytotoxicity by inhibiting $\mathrm{NO}$ release by macrophages (Maeda et al., 2013).

Despite advances in the production of genetically modified animals, inheritance and expression instability of the transgene are still major limitations (Kong et al., 2009; Robertson et al., 1996; Kearns et al., 2000). In many instances, the transgene expression level declines with time and may be completely silenced after several generations (Haruyama et al., 2009; Kong et al., 2009; Robertson et al., 1996). It was shown that the expression of transgene is associated with copy number and DNA methylation in transgenic pigs (Kong et al., 2009). Moreover, random integration can lead to transgene silencing (Clark et al., 1994). Therefore, comprehensive characterization of transgenic organisms is a main prerequisite for the production of a new line of transgenic animals. Evaluation of transgenic animals should include an assessment of transgene integration, karyotype evaluation, transgene expression level, copy number and the functional effect of transgene expression. The aim of this study was to prepare an expressive pHLAE-GFPBsd gene construct containing the human gene-encoding HLA-E antigen under the promoter of EF-1 $\alpha$ elongation factor, ensuring systemic expression and introduction by microinjection into a pronucleus of the fertilized porcine oocytes. In this study, we present the results of a detailed characterization of three generations of transgenic pigs with HLA-E expression using molecular, cytogenetic and functional approaches. 


\section{Material and methods}

\section{Gene construct}

The pHLAE-GFPBsd gene construct was obtained by introducing a cDNA encoding HLA-E antigen (1195 bp) into a pTracer-EF/Bsd A (5987 bp) plasmid containing a strong, constitutive promoter of $1 \alpha$ subunit of human elongation factor EF (EF-1 $\alpha$ ) ensuring systemic expression and a poly(A) sequence of the cattle GH (grow hormone) gene. The coding sequence was amplified on a single-strand cDNA template synthesised by oligo(dT) 20 primers and the SuperScript VILO cDNA Synthesis Kit (Invitrogen). Total RNA was isolated from human white blood cells from peripheral blood with the method based on guanidine isothiocyanate (Chomczynski and Sacchi, 1987). Modified primers were used for amplification. Additionally, a sequence recognised by the EcoRI enzyme (5'-TTA GAA TTC GCT GGG ATC ATG GTA GAT GG-3') was placed in the HLAE-F primer and a sequence recognised by the XbaI enzyme (5'-TTA TCT AGA GGC AGC TGT CTC AGG CTT TA-3) was placed in the HLAE-R primer. The polymerase chain reaction (PCR) of $25 \mu 1$ was conducted in the following conditions: $94^{\circ} \mathrm{C}, 60 \mathrm{~s} ; 58^{\circ} \mathrm{C}, 60 \mathrm{~s} ; 72^{\circ} \mathrm{C}, 90 \mathrm{~s} ; 35$ cycles. The reaction mixture contained $1 \mu \mathrm{l}$ of cDNA, $10 \mathrm{pM}$ of primers, $7.5 \mathrm{nM}$ dNTP, $1 \times$ buffer and $1 \mathrm{U}$ of DNA Taq polymerase (Sigma). The PCR product was digested by EcoRI (Fermentas) and $\mathrm{XbaI}$ (Fermentas), purified and ligated with the pTracer-EF/ BsdA vector, which was digested with the same endonucleases. The product was sequenced using automated genetic analysers (Applied Biosystems Prism). The construction prepared for microinjection was purified by the Endo Free Plasmid Purification Maxi Kit (Qiagen) and then linearised with the Eam1105I enzyme.

\section{Generation of HLA-E transgenic pigs}

Exogenous DNA as a pHLAE-GFPBsd gene construct was inserted into the pronucleus of a fertilised egg cell (928 fertilised egg cells from 81 donors were used). Microinjection was performed with the use of two micromanipulators under a reverse microscope with differential interference contrast. The magnification applied was objective $40 \times \mathrm{X}$ eyepiece $=450 \times$. DNA was injected into the pronucleus with the use of an injector or a syringe connected to the holder for a manipulator. The volume of the DNA inserted was 2-5 pl. DNA was inserted until the moment when the pronucleus increased its volume by $50 \%$. The DNA concentration was $2-4 \mathrm{ng} / \mu \mathrm{l}$. After the injection, the damaged zygotes were removed. Good-quality embryos were transplanted into the fallopian tubes of 37 synchronised recipients. From 14 recipients, 86 piglets were obtained in the F0 generation, which were subjected to DNA analysis in order to detect the transgene by PCR. The F1 and F2 generations of genetically modified pigs were obtained by interbreeding.

\section{Screening of transgene}

The analysis included genomic DNA isolation from biopsies of potentially transgenic animals with the use of proteinase K. PCR was conducted on a DNA template. Two pairs of primers were used for amplification. Both F primers were complementary to the hEF-1 $\alpha$ promoter sequence, and both $\mathrm{R}$ primers were complementary to 
the sequence of HLA-E gene. A PCR product of $333 \mathrm{bp}$ was formed with the use of the first pair of HLAE-F1 (5'-CCAGCTTGGCACTTGATGTA-3') and HLAE-R1 (5'-CACGTAGCCCACAGAGATGA -3') primers, whereas a second product of 481 bp was formed with the use of the F2 ( $5^{\prime}$-GGACACGGAAGTGTGGAAAT-3') and R2 (5'-GGACACGGAAGTGTGGAAAT-3') primers. PCR was conducted in $20 \mu \mathrm{l}$ in the following conditions: $94^{\circ} \mathrm{C}, 40 \mathrm{~s} ; 60^{\circ} \mathrm{C}, 30 \mathrm{~s} ; 72^{\circ} \mathrm{C}, 60 \mathrm{~s} ; 35$ cycles. The reaction contained $100 \mathrm{ng}$ of genomic DNA, $10 \mathrm{pM}$ of primers, $7.5 \mathrm{nM}$ of dNTP, $1 \times$ buffer and $1 \mathrm{U}$ of DNA Taq polymerase (Sigma Aldrich). Simultaneously, to check the quality of the DNA, the 361-bp DNA fragment was amplified using primer SUSF1 (5'-TGTGTGACGAAGACGAGACC-3') and primer SUS-R1 (5'-CTTCTCCATGTCGTCCCAGT-3’).

\section{Cell culture}

Primary fibroblast cell lines were started from ear biopsy specimens of pigs. Ear biopsy specimens collected in sterile conditions were placed in a Phosphate Buffered Saline (PBS) solution containing an antibiotic/antimycotic solution (Antibiotic Antimycotic Solution for Cell Culture, Sigma Aldrich). Sterile tissues were rinsed in a Hank's solution and cut into small pieces. Skin fragments were suspended in Dulbecco's Modified Eagle Medium (DMEM, Sigma-Aldrich) supplemented with $20 \%$ fetal bovine serum (FBS, Sigma-Aldrich), 1\% antibiotic/antimycotic solution and cultivated at $37^{\circ} \mathrm{C}$ in a humidified atmosphere containing $5 \% \mathrm{CO}_{2}$ in $25 \mathrm{~cm}^{2}$ culture flasks. The culture was established in sterile conditions, using a cabinet with a laminar air flow (Heraeus, Germany). The medium was replaced every 3 days. Cultures of $80 \%$ confluence were split 1:2 in a fresh medium and placed into new culture flasks. The cell lines used in experiments did not exceed 6 passages.

Primary porcine aortic endothelial cells were established in our laboratory and were isolated from modified and wild-type pigs. In order to isolate endothelial cells $(8-10 \mathrm{~cm})$ fragments of aorta $(8-10 \mathrm{~cm})$ were washed 3 times in PBS. Then the pieces were placed on a sterile tray. The ends of the aorta were spun with umbilical cord clips. The aorta was filled with collagenase IV through the artery intercostal hole and placed at $37^{\circ} \mathrm{C}$ (incubator) for 10 minutes. After incubation, the cell suspension inside the aorta was transferred to a $15 \mathrm{ml}$ centrifuge tube. Additionally, remaining endothelial cells in aorta were scraped with a sterile swab and rinsed with additional collagenase. $5 \mathrm{ml}$ of a previously prepared culture medium for endothelial cells (RPMI, 10\% FBS (Fetal Bovine Serum), 1\% antibiotic, endothelial cell growth factor, heparin) was added to the cell suspension and centrifuged at $9000 \mathrm{rcf}, 5$ minutes at room temperature. After centrifugation, the supernatant was removed and the cells were resuspended in $3 \mathrm{ml}$ of the culture medium. Cells were transferred to 6 well culture plates coated with $0.1 \%$ gelatin. The culture plate was placed at $37^{\circ} \mathrm{C}$, $5 \% \mathrm{CO}_{2}$ atmosphere (incubator). After 24 hours, medium was replaced. After 72 hours, the cells were moved and grown in $25 \mathrm{~cm}^{2}$ culture vessels. The cells were cultured until they reached $80-90 \%$ confluency. After reaching the proper confluence, cell passage was performed.

A NK92MI (ATCC), interleukin-2 independent human natural killer cell line was cultured in an Alpha Minimum Essential medium supplemented with $2 \mathrm{mM}$ L-glutamine, $1.5 \mathrm{~g} / \mathrm{L}$ sodium bicarbonate, $0.2 \mathrm{mM}$ inositol, $0.1 \mathrm{mM}$ 2-mercaptoetha- 
nol, $0.02 \mathrm{mM}$ folic acid, horse serum to a final concentration of $12.5 \%$, fetal bovine serum to a final concentration of $12.5 \%$ and $1 \%$ antibiotic/antimycotic. The cultures were maintained by centrifuging cells and resuspending cell pellets in a fresh medium at $2-3 \times 10^{5}$ viable cells $/ \mathrm{mL}$.

\section{Obtaining metaphase preparations}

To arrest the cell division of porcine fibroblasts in a mitotic metaphase by means of damaging the spindle apparatus, colcemid $(0.05 \mu \mathrm{g} / \mathrm{ml})$ (Sigma-Aldrich) was added to the culture dishes of $80 \%$ confluence and the cells were incubated at $37^{\circ} \mathrm{C}$ for $3 \mathrm{~h}$. The cells were then subjected to osmotic shock by being suspended in a $75 \mathrm{mM}$ $\mathrm{KCl}$ solution. After 30 min incubation, the material was fixed with a cold mixture of methanol and acetic acid (3:1) three times. The number and dispersion of metaphase plates was evaluated during observation with a Nikon Eclipse E400 optical microscope in phase contrast mode.

\section{FISH analysis}

A molecular probe (pHLAE-GFPBsd) was directly labeled with fluorescein isothiocyanate (FITC) in random priming. Next, fluorescence in situ hybridization (FISH) was conducted. The metaphase chromosomes obtained earlier were digested with RNase (final concentration of $100 \mu \mathrm{g} / \mathrm{ml}$ in a $2 \times \mathrm{SSC}$ solution), washed $(2 \times \mathrm{SSC}, \mathrm{PBS})$ and dehydrated in alcohols in order of their increasing strength. Then, denaturation of slides on a heating panel was carried out at $80^{\circ} \mathrm{C}$ for $30 \mathrm{~min}$ and next in $70 \%$ formamide $/ 2 \times \mathrm{SSC}$ at $70^{\circ} \mathrm{C}$ for $2 \mathrm{~min}$. Probes were denaturated at $70^{\circ} \mathrm{C}$ for $10 \mathrm{~min}$. For hybridization, slides with a probe were incubated overnight in a humid environment at $37^{\circ} \mathrm{C}$. The following day, the slides were washed three times in $50 \%$ formamide $/ 2 \times \mathrm{SSC}$ at $42^{\circ} \mathrm{C}$ for $5 \mathrm{~min}$, three times in $2 \times \mathrm{SSC}$ at $42^{\circ} \mathrm{C}$ and placed in $0.1 \%$ Tween $20 / 4 \times \mathrm{SSC}$ at room temperature $(\mathrm{RT})$ for $10 \mathrm{~min}$. In order to enable observation under a fluorescent microscope, DAPI/Antifade was used for staining. Analysis of the hybridization signal was performed with the use of a microscope with a camera (Axio Imager M.2, Carl Zeiss Microscopy GmbH, Germany) and appropriate software (MetaSystems).

\section{Karyotype analysis}

Karyotype evaluation in the animals was based on the GTC band pattern obtained and on available patterns of domestic pig karyotypes (Gustavsson, 1988). It involved the trypsinisation of chromosome preparations and their being stained with a Giemsa solution. In order to arrange the chromosomes in homologous pairs and chromosome groups corresponding to the pattern, the resulting chromosome bands were analysed using a microscope with a camera (Axio Imager M.2, Carl Zeiss Microscopy GmbH, Germany) and appropriate software (Ikaros).

\section{mRNA expression analysis of transgene}

Expression of the HLA-E mRNA in skin fibroblasts obtained from transgenic pigs was analysed by reverse-transcription PCR (RT-PCR). Total RNA was isolated from porcine fibroblasts using the Total RNA Mini Plus Kit (A\&A Biotechnology). The 
detection of HLA-E mRNA was carried out by a reverse transcriptase reaction using the SuperScript VILO cDNA Synthesis Kit (Invitrogen). For PCR, $1 \mu 1$ of cDNA solution was used. PCR was performed using 35 cycles (primer annealing $56^{\circ} \mathrm{C}$; extension $72^{\circ} \mathrm{C}$; denaturation $94^{\circ} \mathrm{C}$ ). The $1195 \mathrm{bp}$ cDNA fragment was amplified using primer HLAE-F (5' -TTAGAATTCGCTGGGATCATGGTAGATGG-3') and primer HLAE-R (5'-TTATCTAGAGGCAGCTGTCTCAGGCTTTA-3'). Simultaneously, to check the quality of the cDNA, the 319-bp cDNA fragment was amplified using primer bactin-F (5'-ACATCAAGGAGAAGCTGTGCTAC-3') and primer bactinR (5'-CTTCATGATGGAGTTGAAGG TAGTT-3'). The PCR products were separated on a $1.5 \%$ agarose gel.

The expression level of the transgene in skin fibroblasts obtained from transgenic pigs was analysed by real-time PCR with SYBR green dye. A real-time PCR reaction was performed employing KAPA SYBR FAST One-Step qRT-PCR Kit Universal (KAPA Biosystems) and Mastercycler ep realplex apparatus (Eppendorf). The real-time PCR reaction was conducted under the following conditions: $42^{\circ} \mathrm{C}$, $5 \mathrm{~min} ; 95^{\circ} \mathrm{C}, 5 \mathrm{~min} ; 95^{\circ} \mathrm{C}, 15 \mathrm{sec} ; 59^{\circ} \mathrm{C}, 20 \mathrm{sec} ; 72^{\circ} \mathrm{C}, 20 \mathrm{sec} ; 30$ cycles. The eHLA-F5 (5'-TTCCGAGTGAATCTGCGGAC-3') and eHLA-R5 (5'-AGGCGAACTGTTCATACCCG-3') primers were used to amplify a $133 \mathrm{bp}$ fragment of cDNA for the HLA-E gene. In order to quantify the relative levels of transgene expression, the GAPDH and $\beta$-actin were used as reference genes. Bactin-F and bactin-R primers were used to amplify a fragment of cDNA for the $\beta$-actin gene and GAPDH-F1 (5'-GGGCATGAACCATGAGAAGT-3') and GAPDH-R1 (5'-TGTGGTCATGAGTCCTTCCA-3') primers were used to amplify a $133 \mathrm{bp}$ fragment of cDNA for the GAPDH gene. In order to minimize the error associated with the different quantity of the template applied, the analysis was repeated three times and the results obtained were averaged. The results were analysed with the assistance of Realplex Software version 2.2.

\section{Flow cytometry}

Having reached full confluence, the skin fibroblasts cultivated in vitro were washed with Hank's solution and trypsinised (0.25\% trypsin, 0.02\% EDTA). Fibroblasts were collected by centrifugation. The cells were suspended and incubated in PBS containing phycoerythrin (PE)-conjugated antibody (PE anti-human HLA-E, BioLegend) $(40 \mu \mathrm{g} / \mathrm{ml})$, at $4{ }^{\circ} \mathrm{C}$, for $30 \mathrm{~min}$. A BD FACS Aria ${ }^{\mathrm{TM}}$ III (Becton Dickinson) flow cytometer with four lasers $(375,405,488$ and $633 \mathrm{~nm})$, eleven detectors of fluorescence and dispersed laser light detectors-forward scatter and side scatter were used for the analysis of the HLA-E antigen on the surface of the cells. Optical alignment and functional stability tests were conducted with the CST system (Cytometer Setup and Tracking; Becton Dickinson). The data were analysed with the use of FACS DIVA software (Becton Dickinson). The levels of HLA-E antigen on the surface of the cells were determined based on the median values of fluorescence signals. Each sample was analysed three times.

Determination of the absolute number of transgene copies in transgenic pigs

The copy number of the transgene that had integrated with the genome of the transgenic animals was analysed by real-time PCR with SYBR green dye, which 
enabled nonspecific double-stranded DNA detection. The real-time PCR reaction was performed by means of KAPA SYBR FAST qPCR Kit Master Mix (2x) Universal (KAPA Biosystems) and the Mastercycler ep realplex apparatus (Eppendorf). HLAE-F3 (5'-TGGAATTTGCCCTTTTTGAG-3') and HLAE-R3 (5'-GGTCCTCATTCAGGGTGAGA-3') primers were used to amplify a 609 bp fragment of DNA. The forward primer had a sequence complementary to a promoter sequence and reverse primer to a coding sequence of HLA-E antigen. DNA, used as a template, was isolated from the animal peripheral blood employing the method with guanidine isothiocyanate. The real-time PCR reaction was conducted under the following conditions: $95^{\circ} \mathrm{C}, 5 \mathrm{~min} ; 95^{\circ} \mathrm{C}, 15 \mathrm{sec} ; 58^{\circ} \mathrm{C}, 20 \mathrm{sec} ; 72^{\circ} \mathrm{C}, 20 \mathrm{sec} ; 32 \mathrm{cycles}$.

The copy number of the transgene in the samples was established by plotting the $\mathrm{Ct}$ (cycle threshold) values obtained on a standard curve, which had been set by the usage of the ct values for the successive standard dilutions with a known copy number $\left(1.3^{8}-1.3^{3}\right.$ copies $)$. As a standard we used a pHLAE-GFPBsd genetic construct hydrolyzed with a $S c a$ I restriction enzyme to a linear form. In order to minimize the error associated with the different quantity of the template, the analysis was repeated three times and the results were averaged. These results were analysed with the assistance of Realplex Software version 2.2.

\section{Cytotoxicity test}

To evaluate the suppressive effect of HLA-E expression on porcine endothelial cells against human NK cells cytotoxicity, an IL-2-independent natural killer (NK) cell line NK-92MI (ATCC) was cocultured with endothelial cells derived from HLAE transgenic animals or with wild-type endothelial cells as controls. $100 \mu \mathrm{l}$ of endothelial cell suspension (5000 cells/well) was dispensed in a 96-well plate as target cells. After pre-incubation for $24 \mathrm{~h}$ in complete media (DMEM, 10\% FBS, 1\% antibiotic/ antimycotic) in a humidified incubator (at $37^{\circ} \mathrm{C}, 5 \% \mathrm{CO}_{2}$ ), the media were removed and human NK cells (effector cells) were added to the target cells. 5:1 effector cell to target cell $(\mathrm{E}: \mathrm{T})$ ratios were used in each experiment. These cells were cocultured for $4 \mathrm{~h}$ in a complete growth medium for NK cells (MEM with $2 \mathrm{mM}$ L-glutamine, $1.5 \mathrm{~g} / \mathrm{L}$ sodium bicarbonate, $0.2 \mathrm{mM}$ inositol, $0.1 \mathrm{mM}$ 2-mercaptoethanol, $0.02 \mathrm{mM}$ folic acid, $12.5 \%$ horse serum, $12.5 \%$ fetal bovine serum). The cytotoxic activity of the human NK cell lines NK92MI was tested in sensitive colorimetric assays using a Cell Counting Kit-8 (Sigma Aldrich). Cell Counting Kit-8 (CCK-8) allows convenient assays using WST-8 (2-(2-methoxy-4-nitrophenyl)-3-(4-nitrophenyl)-5-(2,4disulfophenyl)-2H-tetrazolium, monosodium salt), which produces a water-soluble formazan dye upon bioreduction in the presence of an electron carrier. WST-8 is reduced by cellular dehydrogenases to an orange formazan product that is soluble in the tissue culture medium. The amount of formazan produced is directly proportional to the number of living cells. Cell Counting Kit-8 allows sensitive colorimetric assays for determining the number of viable cells in the cytotoxicity assays. After $4 \mathrm{~h}$ incubation, $10 \mu \mathrm{L}$ of the CCK-8 reagent was added to each well, and the cells were incubated for $2 \mathrm{~h}$ at $37^{\circ} \mathrm{C}$. The spectrophotometric absorbance of each sample was measured using a microplate reader at $450 \mathrm{~nm}$. The percentage of surviving cells in each test group was calculated. These experiments were carried out three times. 


\section{Results}

\section{Generation of transgenic pigs with HLA-E gene}

The pHLAE-GFPBsd gene construct (Figure 1) was obtained by introducing cDNA coding for HLA-E into pTracer-EF/Bsd A plasmid and was introduced by microinjection into one of the pronuclei of the zygote. The PCR analysis showed that one female piglet in the F0 generation (TG1151) incorporated the transgene (1/86; $1.16 \%)$. When the female TG1151 reached puberty, she was interbred. As a result of interbreeding, 10 piglets were obtained, of which there was 1 sow (TG579) with the HLA-E gene $(1 / 10 ; 10 \%)$. In the next step, the individual TG579 was interbred and 11 live piglets were obtained. The piglets in the F2 generation included two sows (TG102, TG109) and four boars (TG105, TG106, TG107, TG110) with confirmed integration of the pHLAE-GFPBsdb (Figure 2).

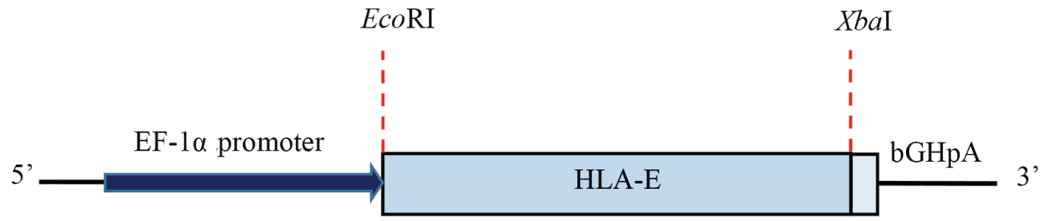

Figure 1. The scheme shows the pHLAE-GFPBsd gene construct encompassing a strong, constitutive EF-1 $\alpha$ promoter, cDNA of the gene coding for HLA-E gene and the poly(A) sequence of the bovine GH gene. The scheme also shows the locations of the restriction sites for EcoRI and XbaI enzymes

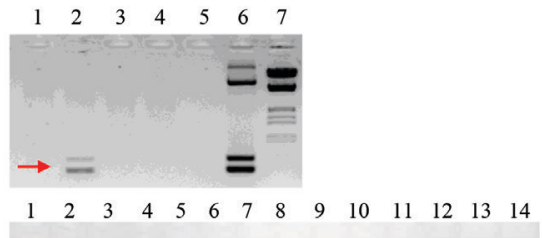

B

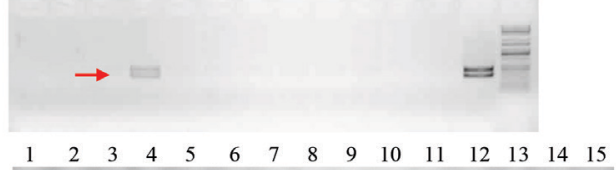

C

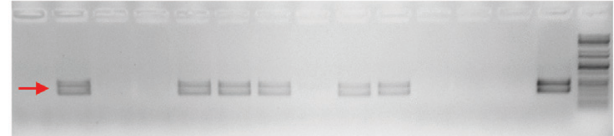

Figure 2. Analysis of the integration pHLAE-GFPBsd with genomic DNA in pigs (F0, F1, F2 generations). Polymerase chain reaction (PCR) was used to amplify DNA fragments of $333 \mathrm{bp}$ and $481 \mathrm{bp}$. (A) Lanes 1-3, DNA isolated from TG1150, TG1151, TG1152; lane 4, negative control (wild-type pig DNA); lane 5, negative control (without DNA); lane 6, positive control (pHLAE-GFPBsd construct); lane 7, size marker ( $\lambda$ DNA/HindIII, EcoRI). (B) Lanes 1-10, DNA isolated from TG576 - TG585; lane 11, negative control (wild-type pig DNA); lane 12, negative control (without DNA); lane 13, positive control (pHLAE-GFPBsd construct); lane 14, size marker (KAPA Universal Ladder Kits). (C) Lanes 1-11, DNA isolated from TG101 - TG111; lane 12, negative control (wild-type pig DNA); lane 13, negative control (without DNA); lane 14, positive control (pHLAE-GFPBsd construct); lane 15, size marker (KAPA Universal Ladder Kits). The separation of DNA fragments was conducted in 1.5\% agarose gel. The red arrows indicate transgenic piglet 


\section{Cytogenetic analysis}

Integration analysis carried out by the PCR method was confirmed by fluorescence in situ hybridization in all transgenic animals with the HLA-E gene. FISH was conducted with the use of molecular probes complementary to the pHLAE-GFPBsd plasmid. In all transgenic pigs (TG1151, TG579, TG102, TG105, TG106, TG107, TG109, TG110), HLA-E transgene was detected in a heterozygous arrangement on one of the metacentric chromosomes. The example result of FISH analysis is shown in Figure 3. Karyotype evaluation in transgenic individuals with the HLA-E gene was carried out by staining the digested chromosomes with a Giemsa stain. The resulting band pattern of chromosomes was compared with the standard karyotype of the domestic pig. Karyotype analysis did not show any changes in the structure or the number of chromosomes. The correct karyotypes of TG1151 $(2 \mathrm{n}=38, \mathrm{XX})$ and TG106 $(2 \mathrm{n}=38, \mathrm{XY})$ are shown in Figure 4.

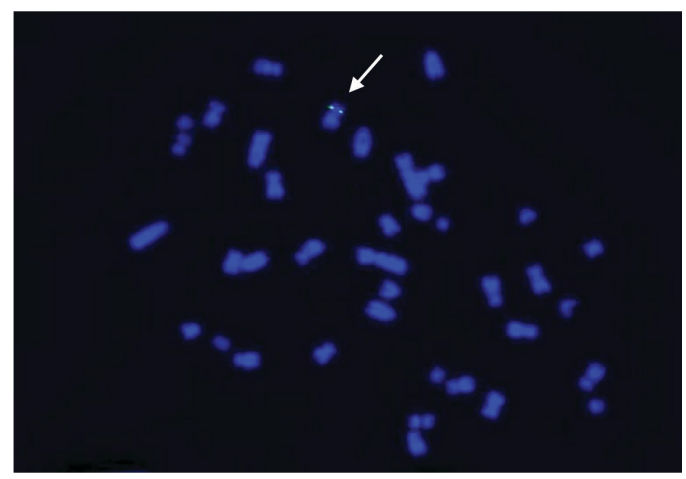

Figure 3. Confirmation of the presence of pHLAE-GFPBsd construct in transgenic pig by FISH analysis. Post-hybridisation image of metaphase chromosomes from a cell line derived from skin fibroblasts of TG107. Molecular probe complementary to plasmid pHLAE-GFPBsd (probe labeled with FITC - color green). Chromosomes stained with DAPI- blue. The white arrow indicates heterozygous configuration of transgene

A

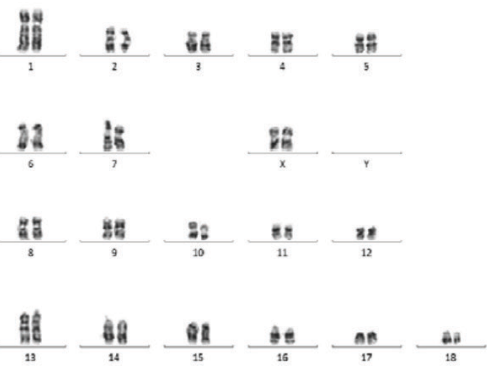

B

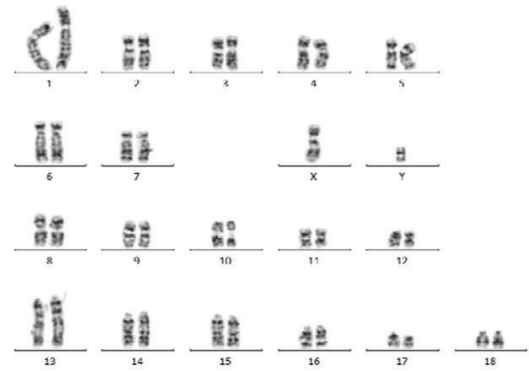

Figure 4. Karyotype analysis in transgenic pigs with HLA-E gene. (A) Correct karyotype of a pig from the F0 generation, TG1151 (38, XX). (B) Correct karyotype of a pig from the F2 generation, TG106 $(38, \mathrm{XY})$ 


\section{Analysis of transgene expression}

Transgene expression was evaluated by RT-PCR and flow cytometry. The results of RT-PCR showed the expression of the HLA-E mRNA in fibroblasts obtained from TG1151 (F0), TG579 (F1), TG102, TG105, TG106, TG107, TG109 and TG110 (F2) transgenic pigs (Figure 5). Flow cytometry was used to check whether transgene expression results in the presence of the HLA-E antigen on the surface of cells in pigs. The analysis was conducted on fibroblast lines derived from wild-type animals and from all transgenic pigs with the HLA-E gene (F0, F1, F2 generations). Staining with $P E$ anti-human $H L A-E$ showed the presence of the HLA-E antigen in all transgenic animals analysed. The results of statistical analysis showed that the higher intensity of fluorescence signals for fibroblasts from genetically modified pigs compared to the controls was statistically significant (Table 1). The Student's t-test was used for statistical analysis.

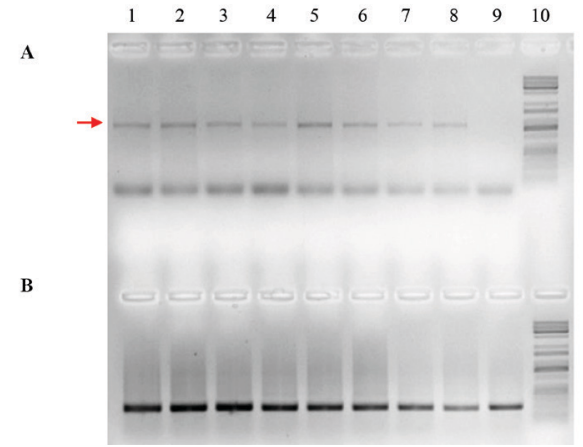

Figure 5. Detection of the HLA-E mRNA expression in fibroblast derived from transgenic pigs. RT-PCR amplification followed by agarose gel electrophoresis. (A) Analysis of pHLAE-GFPBsd expression. RTPCR was used to amplify DNA fragments of $1195 \mathrm{bp}$. (B) Analysis of $\beta$-actin expression (cDNA quality control). Lanes 1-8, TG1151, TG579, TG102, TG105, TG106, TG107, TG109, TG110; lane 9, negative control (wild-type pig); lane 10, size marker (KAPA Universal Ladder Kits). The red arrow indicates HLA-E expression

Table 1. Results of transgene expression analysis by flow cytometry. Statistical analysis of the results confirmed that higher levels of fluorescence of fibroblasts derived from transgenic pigs labeled with

$P E$ anti-human HLA-E compared to control fibroblasts was statistically significant. SD - standard deviation of the mean. Significance level: $\mathrm{x}-\mathrm{P}<0.05, \mathrm{xx}-\mathrm{P}<0.01, \mathrm{xxx}-\mathrm{P}<0.001$

\begin{tabular}{|c|c|c|c|}
\hline Pigs & $\begin{array}{c}\text { Fluorescence level } \\
\text { (mean of three repetitions) }\end{array}$ & SD & Significance level \\
\hline $\begin{array}{c}\text { NT14 } \\
\text { (wild-type pig) }\end{array}$ & 87.3 & 3.33 & \\
\hline TG1151 & 406.0 & 10.97 & $\mathrm{xxx}$ \\
\hline TG579 & 401.0 & 11.53 & $\mathrm{xxx}$ \\
\hline TG106 & 306.3 & 5.24 & $\mathrm{xxx}$ \\
\hline TG109 & 366.7 & 13.84 & $\mathrm{xxx}$ \\
\hline $\begin{array}{l}\text { NT14 } \\
\text { (wild-type pig) }\end{array}$ & 86.3 & 2.33 & \\
\hline TG102 & 367.3 & 29.80 & $\mathrm{x}$ \\
\hline TG105 & 303.3 & 15.30 & $\mathrm{xx}$ \\
\hline TG107 & 354.3 & 6.17 & $\mathrm{xxx}$ \\
\hline TG110 & 337.7 & 12.88 & $\mathrm{xxx}$ \\
\hline
\end{tabular}


Moreover, we introduced a real-time PCR method for quantitative determination of the transgene expression level in skin fibroblast derived from transgenic pigs with the HLA-E gene. The relative level of transgene expression of the F1 and F2 generations of genetically modified pigs was calibrated to the founder HLA-E transgenic pig (F0), for which the level of transgene expression was taken as 1 . The Student's t-test was used for statistical analysis. The results of real-time PCR analysis allowed confirmation that the expression level of the pHLAE-GFPBsd transgene was stable in the next generation of genetically modified pigs (Table 2).

Table 2. Results of relative real-time PCR analysis of HLA-E expression. The level of HLA-E expression in offspring (generations F1 and F2) was calculated in relation to expression in founder pig TG1151 (generation F0) for which expression was assumed as 1. The transgene silencing was not observed

\begin{tabular}{c|c|c|c}
\hline Transgenic pigs & Generation & $\begin{array}{c}\text { Level of transgene expression } \\
\text { (mean of six repetitions) }\end{array}$ & SD \\
\hline TG1151 & F0 & 1 & - \\
TG579 & F1 & 1.695 & 0.12 \\
TG102 & F2 & 1.863 & 0.37 \\
TG105 & F2 & 1.297 & 0.21 \\
TG106 & F2 & 1.309 & 0.27 \\
TG107 & F2 & 1.376 & 0.20 \\
TG109 & F2 & 3.297 & 0.32 \\
TG110 & F2 & 1.325 & 0.27 \\
\hline
\end{tabular}

\section{Transgene copy number}

Since $1 \mathrm{ng}$ of DNA corresponds to the molecular mass of $602000000 \mathrm{MDa}$ and the molecular mass of the swine genome amounts to $1817200 \mathrm{MDa}, 1 \mathrm{ng}$ of swine genomic DNA contains approximately 331.3 copies of the genome. $30 \mathrm{ng}$ of swine genomic DNA sample was used, hence each of the samples contained about 9939 copies of the swine genomic DNA. In order to calculate the number of copies of the transgene incorporated into a single genome of transgenic animals, the absolute number of copies of the transgene in titration samples was divided by the number of copies of the genomic DNA. In the case of heterozygotic transgenic animals, the transgene only occurs on one of the pair of homologue chromosomes, therefore in order to calculate the number of transgene copies built into one of the chromosomes, the results of calculations were multiplied by 2 . The results of real-time PCR analysis allowed the number of pHLAE-GFPBsd transgene copies incorporated into the genome of transgenic pigs at the interval of 16 to 34 to be estimated (Table 3 ). As expected, no amplification of the PCR product was observed in the case of wild-type animals.

\section{Cytotoxicity test}

The functional effect of transgene expression on endothelial cells derived from HLA-E transgenic pigs was tested in NK cell cytotoxicity assays using wild-type endothelial cells as controls. The survival rate was determined by CCK-8 colorimetric 
assays for the determination of cell viability in cell cytotoxicity assays. The results of absorbance measurement cells after incubation with human NK cells led to the conclusion that higher cell viability occurred in the case of HLA-E expressing target cells (survival rate $88.45 \%$ ) compared to wild type (70.42\%) (Figure 6). The Welch Two Sample t-test was used for statistical analysis.

Table 3. Results of absolute quantitative real-time PCR analysis of HLA-E copy number. The number of pHLAE-GFPBsd transgene copies incorporated into the genome of TG1151, TG579, TG102,

TG105, TG106, TG107, TG109 and TG110 transgenic pigs was estimated at the interval of 16 to 34

\begin{tabular}{c|c|c|c}
\hline Transgenic pigs & Generation & Transgene copy number & SD \\
\hline TG1151 & F0 & 16.6 & 3.6 \\
TG579 & F1 & 16.2 & 7.5 \\
TG102 & F2 & 22.2 & 4.6 \\
TG105 & F2 & 30.1 & 4.4 \\
TG106 & F2 & 22.4 & 8.4 \\
TG107 & F2 & 33.8 & 8.5 \\
TG109 & F2 & 32.6 & 13.1 \\
TG110 & F2 & 23.7 & 6.0 \\
\hline
\end{tabular}

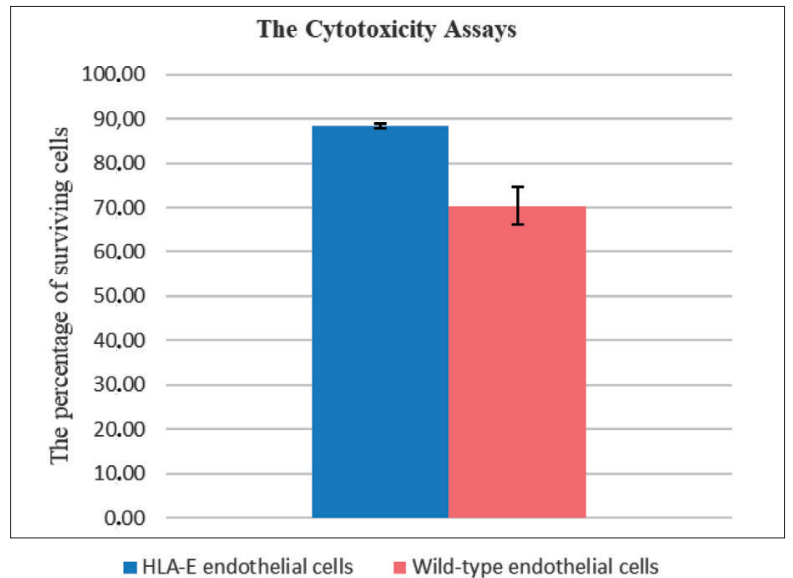

Figure 6. Survival rate analysis for cells from HLA-E transgenic pig and wild type pig in the medium with human NK cells. After co-culture for $4 \mathrm{~h}, \mathrm{CCK}-8$ was used to detect the remaining vital cells. The percentage of surviving cells in each tested group was marked on Y axis. The higher cell viability occurred in the case of HLA-E expressing cells (survival rate $88.45 \%$ ) compared to wild type $(70.42 \%)$. The probability value $=0.01639(\mathrm{P}<0.05)$

\section{Discussion}

Organ transplant in the xenogenic pig-human system may solve many problems of contemporary transplantology. One of the challenges faced by xenotransplantology is connected with the generation of transgenic pigs, whose cells, tissues and 
organs may be resistant to acute and delayed xenotransplant rejection. This paper presents how a gene construct is obtained with a sequence coding the human leukocyte antigen-E. This strategy aims to inhibit xenotransplant rejection resulting from the cytotoxic action of human NK cells against porcine endothelial cells. It was shown that NK cells may penetrate pig organs with human blood ex vivo and cause lysis of porcine endothelial cells in vitro (Itescu et al., 1996; Khalfoun et al., 2000). CD94/NKG2A lectin receptors of human NK cells mediate the inhibition of NK cell activation. The CD94/NKG2A receptors are capable of binding HLA-E and transmitting signals blocking cell activation. It was shown that the expression of the HLA-E gene into the porcine cells partially protects the recipient against human NK cells (Lilienfeld et al., 2007). Bongoni and co-workers introduced the HLA-E gene together with the human CD46 gene (CD46, membrane cofactor protein) into the pig genome. These modifications significantly inhibited the humoral response, complement and endothelial activation, as well as proinflammatory cytokine production in an ex vivo model of transgenic pig limbs perfused with human blood (Bongoni et al., 2014). Laird and co-workers directly compared GTKO/hCD46/HLA-E (GTKO, knockout of the galactosyl transferase enzyme) pig lungs to GTKO/hCD46 reference organs and indicated that additional expression of the HLA-E gene increased median lung survival when perfused ex vivo with human blood and was associated with diminished histamine elaboration and platelet activation (Laird et al., 2017). In this study, the pHLAE-GFPBsd gene construct was obtained by the insertion of the cDNA sequence of the HLA-E gene into a pTracer-EF/Bsd A plasmid vector containing a strong, constitutive EF- $1 \alpha$ promoter. EF- $1 \alpha$ is active in a broad range of cell types and is often a useful promoter in conditions where other promoters, such as cytomegalovirus (CMV), have reduced activity or have been gradually silenced (Norrman et al., 2010; Grassi et al., 2003). In order to increase the stability of mRNA of the inserted gene, the polyadenylation (pA) signal of the bovine growth hormone (BGH) gene was used. The gene construct was introduced into the pig genome by microinjection into one of the pronuclei of a fertilized egg cell. The first to obtain a transgenic pig with the HLA-E gene was Weiss et al. (2009), using SCNT. SCNT is thought to be limited by a high rate of embryonic loss, elevated risk of generating abnormal animals and low efficiency of producing offspring (Petersen et al., 2008; Wang et al., 2015). Therefore, we decided to adopt direct microinjection of the pHLAE-GFPBsd gene construct into the pronuclei of zygotes. Although our efficiency of transgenic pig production was also low (one F0 founder out of 86 piglets), we were able to obtain transgenic animals by crossbreeding. PCR was used to facilitate a simple and rapid evaluation of a large number of potentially transgenic animals and the FISH technique was used to confirm transgene integration. The four sows and four boars (F0, F1, F2 generations) with the confirmed presence of HLA-E transgene were then subjected to detailed molecular and cytogenetic analyses, including analyses of the expression of genes introduced into the pig genome, determination of the transgene copy number, as well as the analysis of the karyotype of the transgenic animals. These methods made it possible to verify whether the foreign gene introduced into the pig genome was integrated with the genome in a stable manner and whether the new trait was transmitted from generation to generation. In the 
present study, we used an EF-1 $\alpha$ promoter, which provided a systemic expression of the controlled gene, although its level depends on the transgene insertion site (Kim et al., 1990; Taboit-Dameron et al., 1999). The transgene expression in genetically modified pigs was investigated using RT-PCR to determine gene expression at the mRNA level, as well as flow cytometry to detect proteins coded by the gene introduced into the recipient genome. Both analyses confirmed the transgene expression in all the animals analysed, thus suggesting that transgene integrated with genomic DNA of pigs was incorporated into the transcriptionally active chromatin region of the host. The random integration of an exogenous gene can disrupt the function or regulation of host genes, resulting in chromosomal aneuploidy. Parkanyi et al. (2004) noted a significantly higher rate of aneuploidy in transgenic (56-66\%) rabbits, as compared to non-transgenic ones (28-38\%). Therefore, the next element in the characteristics of transgenic animals in this study was to evaluate the karyotype in terms of the incidence of chromosome aberrations. Our analysis showed no changes in the number and structure of chromosomes in any of the transgenic animals examined. These results allow us to state that the introduction of the transgene into the pig genome by pronuclear microinjection did not have a negative impact on the karyotype of the genetically modified animals that were obtained.

In random introduction of DNA into the genome, the transgene usually integrates in a tandem manner. Most typically, the inserted DNA is integrated with the genome of the recipient as concatamers, i.e. a series of bound transgene molecules, formed as a result of homologous recombination between molecules of exogenous DNA (Folger et al., 1982). Determination of the transgene copy number was the next stage in the molecular characteristics of transgenic animals. The results of real-time PCR showed that the copy number of the pHLAE-GFPBsd transgene in the eight transgenic pigs that were analysed ranged from 16 to 34 . A similar result from analysis of the transgene copy number provided by real-time PCR (between 18.87 and 37.59 transgene copies) was reported by another research team during analysis of F0 transgenic pigs with the introduction of the green fluorescent protein (GFP) gene controlled by the CMV promoter (Kong et al., 2009). Moreover, that team showed that the transgene copy number may vary depending on the type of tissue being studied (analyses were conducted on samples of e.g. uterine, spleen, ovarian, muscular, hepatic, intestinal, lung, kidney, stomach and cardiac tissues). It is suggested that when applying this method it is difficult to determine precisely the number of copies of the inserted gene for a given animal, which may explain the differences in the copy number recorded in this study when analysing transgenic pigs with the HLA-E gene. However, we may not exclude a slight increase in the copy number of the inserted gene thanks to the homologous recombination between transgene molecules in the tandem repeats. When many copies of transgenes are arranged as a concatameric array, the transgene expression may be silenced (Garrick et al., 1998). For this reason it is crucial to control the transgene expression level in each successive generation of transgenic animals. Kearns and co-workers noted reduced transgene expression with female transmission (2000), but in our study, we did not observe transgene silencing in offspring when the exogenous gene was inherited from the mother. In the present 
study, real-time PCR analysis confirmed HLA-E gene expression stability in three consecutive generations of transgenic pigs. Our results indicate stable and consistent inheritance of the transgene. The higher expression level observed in some F1 and F2 animals may have resulted from the fact that the material for analyses comprised cultures of fibroblasts, which may have differed in their proliferation rates and thus also the profile of transcription activity.

Cytotoxicity analysis showed that cells with the HLA-E gene showed greater viability in comparison to the control. Similarly, Weiss and co-workers demonstrated only partial and not complete protection of HLA-E transgenic porcine lymphoblasts against human NK cell cytotoxicity (2009). Only partial inhibition of human NK cell cytotoxicity may be explained by the fact that HLA-E is not the only antigen regulating the activity of NK cells. The cytotoxic effect on pig cells leading to their lysis is also caused by binding with NKG2D activating receptors of the porcine ULBP1 antigen (Forte et al., 2005). It was shown that partial protection against the cytotoxicity of human NK cells was provided by inactivation of the porcine ULBP1 gene (Zeyland et al., 2018). Partial protection against the cytotoxicity of NK cells is also facilitated by the expression of the HLA-Cw3, HLA-Cw4 or HLA-G genes in pig cells (Forte et al., 2000, 2009; Seebach et al., 1997). Thus, it is suggested that to effectively prevent NK cell-dependent xenotransplant rejection it is necessary to apply several genetic modifications simultaneously.

Through data analysis, the comprehensive molecular characterization of the three generations of transgenic pigs was efficiently and accurately verified. All tested transgenic pigs with HLA-E expression showed the genetic stability of the exogenous insertion gene without any abnormalities in karyotype, therefore, these animals could be used to cross with other genetically modified animals in order to derive multitransgenic pigs, whose cells, tissues or organs transplanted into human recipients would be resistant to xenograft rejection.

\section{Acknowledgments}

The authors from the Poznan University of Life Sciences are members of COST Action BM1308 "Sharing Advances on Large Animal Models (SALAAM)". We would like to thank Ph.D. students Natalia Mazurkiewicz and Paweł Śledziński for technical support.

\section{References}

Bongoni A.K., Kiermeir D., Jenni H., Bähr A., Ayares D., Klymiuk N., Wolf E., Voegelin E., Constantines cu M.A., S e eba ch J.D., Ri eben R. (2014). Complement dependent early immunological responses during ex vivo xenoperfusion of hCD46/HLA-E double transgenic pig forelimbs with human blood. Xenotransplantation, 21: 230-243.

Cho m c zy n s k i P., S a c chi N. (1987). Single-step method of RNA isolation by acid guanidinium thiocyanate-phenol-chloroform extraction. Anal. Biochem., 162: 156-159.

Clark A.J., B iss inger P., Bullock D.W., Damak S., Wallace R., Whitelaw C.B., Yull F. (1994). Chromosomal position effects and the modulation of transgene expression. Reprod. Fert. Develop., 6: 589-598. 
Folg e r K.R., Wong E.A., Wahl G., Cape c ch i M.R. (1982). Patterns of integration of DNA microinjectes into cultured mammalian cells: evidence for homologous recombination between injected plasmid DNA molecules. Mol. Cell Biol., 2: 1372-1387.

Forte P., Matter-Reissmann U.B., Strasser M., Schneider M.K., Seebach J.D. (2000). Porcine aortic endothelial cells transfected with HLA-G are partially protected from xenogeneic human NK cytotoxicity. Hum. Immunol., 61: 1066-1073.

Forte P., Lilienfeld B.G., B a umann B.C., S e ebach J.D. (2005). Human NK cytotoxicity against porcine cells is triggered by NKp44 and NKG2D. J. Immunol., 175: 5463-5470.

F o r t e P., B a u m an n B.C., S c h n e id er M.K., S e e b a c h J.D. (2009). HLA-Cw4 expression on porcine endothelial cells reduces cytotoxicity and adhesion mediated by CD158 $\mathrm{a}^{+}$human NK cells. Xenotransplantation, 16: 19-26.

Garrick D., Fiering S., Martin D.I., Whitelaw E. (1998). Repeat-induced gene silencing in mammals. Nat. Genet., 18: 56-59.

Grassi G., Maccaron i P., Meyer R., Ka is er H., D' A mbrosio E., Pas cale E., Gras si M., Kuhn A., Di Nardo P., Kandolf R., Küpper J.H. (2003). Inhibitors of DNA methylation and histone deacetylation activate cytomegalovirus promoter-controlled reporter gene expression in human glioblastoma cell line U87. Carcinogenesis, 24: 1625-1635.

Gustavs s on I. (1988). Standard karyotype of the domestic pig. Committee for the Standardized Karyotype of the Domestic Pig. Hereditas, 109: 151-157.

$\mathrm{H}$ a r u y a m a N., Cho A., K u 1 k a r n i A.B. (2009). Overview: engineering transgenic constructs and mice. Curr. Protoc. Cell Biol., 42: 19.10.

It escu S., Kwiatkowski P., Wang S.F., Blood T., Minanov O.P., Rose S., Mich1 e r R.E. (1996). Circulating human mononuclear cells exhibit augmented lysis of pig endothelium after activation with interleukin 2. Transplantation, 62: 1927-1933.

Ke arns M., Pre is J., M c D on ald M., M orris C., Whitel aw E. (2000). Complex patterns of inheritance of an imprinted murine transgene suggest incomplete germline erasure. Nucleic Acids Res., 28: 3301-3309.

K halfoun B., B arrat D., Wat ier H., M a che t M.C., A rb eille-B ras sart B., Ries s J.G., S a $1 \mathrm{~m}$ on H., G rue 1 Y., B ard o s P., L e bra n c h u Y. (2000). Development of an ex vivo model of pig kidney perfused with human lymphocytes. Analysis of xenogeneic cellular reactions. Surgery, 128: 447-457.

Kim D.W., Uetsuki T., Kaziro Y., Yamaguchi N., Sugano S. (1990). Use of the human elongation factor 1 alpha promoter as a versatile and efficient expression system. Gene, 91: 217-223.

Kong Q., Wu M., Huan Y., Zhang L., Liu H., Bou G., Luo Y., Mu Y., Liu Z. (2009). Transgene expression is associated with copy number and cytomegalovirus promoter methylation in transgenic pigs. PLoS One., 4(8): e6679.

Laird C.T., B urd orf L., Fren ch B.M., Kubicki N., Cheng X., Braile anu G., S un W., O 'N eill N.A., Cimeno A., Parse 11 D., S o E., Bähr A., K1ymiuk N., Phelps C.J., Ay are s D., A zi m z a de h A.M., P i e r s on R.N. (2017). Transgenic expression of human leukocyte antigen-E attenuates GalKO.hCD46 porcine lung xenograft injury. Xenotransplantation, 24: e12294.

Lilienfeld B.G., Crew M.D., Forte P., B a u mann B.C., S e e b a ch J.D. (2007). Transgenic expression of HLA-E single chain trimer protects porcine endothelial cells against human natural killer cell mediated cytotoxicity. Xenotransplantation, 14: 126-134.

Maed a A., Kaw a mura T., U e no T., U su i N., Eguch i H., M i y a gaw a S. (2013). The suppression of inflammatory macrophage-mediated cytotoxicity and proinflammatory cytokine production by transgenic expression of HLA-E. Transpl. Immunol., 29: 76-81.

Norrman K., Fis cher Y., B on nam y B., Wol fhagen S and F., Ravas sard P., S e mb H. (2010). Quantitative comparison of constitutive promoters in human ES cells. PLoS One., 5: e12413.

Parkányi V., Chrenek P., R a fa y J., S üve gová K., Jurcík R., Makarevich A.V., Piv k o J., H e tén y i L., P a le y a n d a R.K. (2004). Aneuploidy in the transgenic rabbit. Folia Biol., 50: 194-199.

Petersen B., Lucas-Hahn A., Oropeza M., Hornen N., Lemme E., Hassel P., 
Que is ser A.L., N i e mann H. (2008). Development and validation of a highly efficient protocol of porcine somatic cloning using preovulatory embryo transfer in peripubertal gilts. Cloning Stem Cells, 10: 355-362.

Prat he r R.S., S h e n M., D a i Y. (2008). Genetically modified pigs for medicine and agriculture. Biotechnol. Genet. Eng. Rev., 25: 245-265.

Puga Yung G., S c hn e i d e r M.K.J., S e e b a c h J.D. (2017). The role of NK cells in pig-to-human xenotransplantation. J. Immunol. Res., 2017: 4627384, doi: 10.1155/2017/4627384.

Robertson G., Garrick D., Wils on M., Martin D.I., Whitelaw E. (1996). Age-dependent silencing of globin transgenes in the mouse. Nucleic Acids Res., 24: 1465-1471.

S e e b a ch J.D., C o m ra ck C., G erm a na S., L e Guern C., S a c h s D.H., D e r S i monian H. (1997) HLA-Cw3 expression on porcine endothelial cells protects against xenogeneic cytotoxicity mediated by a subset of human NK cells. J. Immunol., 159: 3655-3661.

Sullivan J.A., O ett inger H.F., S a ch s D.H., Edge A.S. (1997). Analysis of polymorphism in porcine MHC class I genes: alterations in signals recognized by human cytotoxic lymphocytes. J. Immunol., 159: 2318-2326.

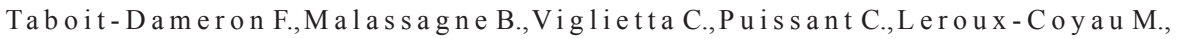
Chéreau C., A ttal J., Weill B., Houd ebine L.M. (1999). Association of the 5'HS4 sequence of the chicken beta-globin locus control region with human EF1 alpha gene promoter induces ubiquitous and high expression of human CD55 and CD59 cDNAs in transgenic rabbits. Transgenic Res., 8: 223-235.

Wang Y., Du Y., Shen B., Zhou X., Li J., Liu Y., Wang J., Zhou J., Hu B., Kang N., G a o J., Yu L., H u a n g X., We i H. (2015). Efficient generation of gene-modified pigs via injection of zygote with Cas9/sgRNA. Sci Rep., 5: 8256.

We is s E.H., Lilienfeld B.G., Muller S., Müller E., Herbach N., Kes sler B., Wanke R., Schwinzer R., S e ebach J.D., Wolf E., B rem G. (2009). HLA-E/human beta2microglobulin transgenic pigs: protection against xenogeneic human anti-pig natural killer cell cytotoxicity. Transplantation, 87: 35-43.

Zeyland J., Hryhorowicz M., Nowak-Terpiłowska A., Jura J., Słomski R., S m or ąg Z., Gajda B., Li pińs ki D. (2018). The production of UL16-binding protein 1 targeted pigs using CRISPR technology. 3 Biotech., 8: 70, doi: 10.1007/s13205-018-1107-4.

Received: 8 III 2018

Accepted: 13 VII 2018 